\title{
EL REVÉS DE LA TRAMA: ELEGIR UNA CARRERA /RECIBIR ESTUDIANTES EN UNA CARRERA.
}

\author{
Analia Umpierrez (UNICEN)*
}

\section{Resumen}

El presente trabajo tiene como antecedente la Tesis de Maestría de la autora en la que se buscó identificar las condiciones y relaciones existentes entre el acceso a una carrera universitaria de profesorado en una de las sedes de una Universidad regional de la Provincia de Buenos Aires, Olavarría, y las elecciones que hacen los sujetos.

En esta elección se tensan y condensan aspectos del plano subjetivo a la vez que del entorno social. Las posibilidades de elección y las oportunidades que se perciben y valoran, respecto de un campo socio-ocupacional, dejan un espacio de juego a los sujetos en los que se define lo que desea, pero en el mismo acto, los limita la propia ubicación del sujeto en un campo social que le muestra "lo posible imaginable" para él/ella. Desde este marco se han seleccionado dos aspectos identificados que invitan a reflexionar y debatir entorno al ingreso a la Universidad Pública. Se propone señalar algunas características identificadas en relación a los componentes de la elección de la carrera de profesorado universitario. Luego, se buscará cambiar de foco e interrogar a la propia institución formadora sobre la percepción construida y compartida respecto de los estudiantes que eligen inscribirse en esas carreras.

\section{Palabras clave}

Elección de carrera - Enseñanza - Ciudad de rango medio - Identidad - Tramas institucionales.

\begin{abstract}
This work came about as a Master Thesis of the author in which we tried to identify the conditions and relationships between access to university faculty on a regional University of the Province of Buenos Aires, one of their headquarters, Olavarria, and the choices made by individuals.
\end{abstract}

Magister en Educación y Licenciada y Profesora de Ciencias de la Educación. Facultad de Ciencias Sociales - Universidad Nacional del Centro de la Provincia de Buenos Aires 
In this election, tense and aspects of subjective level condense while the social environment. Choice and opportunities are perceived and valued in relation to a sociooccupational field, leave a space game to the subjects that define what you want, but at the same ceremony, the limited the very location of the subject in a social field that shows the "possible imaginable" for him / her. From this framework we have chosen two issues identified that invite reflection and discussion around the entrance to the Public University. It is proposed to note some features identified in relation to the components of the choice of university teaching career. Then they seek to change focus and question forming on the institution itself built and shared perceptions on students who choose to enroll in these courses.

\section{Key words}

Choice career - Teaching - Middle size city - Identity - Institutional frames.

\section{Introducción}

El trabajo que aquí se presenta tiene como antecedente la Tesis de Maestría de la autora, en la que se buscó identificar las condiciones y relaciones existentes entre el acceso a una carrera universitaria de profesorado en una Universidad regional de la Provincia de Buenos Aires, en una de sus sedes, Olavarría, y las elecciones que hacen los sujetos.

En esta elección se tensan y condensan aspectos del plano subjetivo a la vez que del entorno social. Las posibilidades de elección y las oportunidades que se perciben y valoran, respecto de un campo ocupacional, dejan un espacio de juego a los sujetos en las que se define lo que quiere, lo que desea, pero en el mismo acto, las limita la propia ubicación del actor en un campo social que le muestra "lo posible imaginable" para sí. Las transformaciones de época, que definen nuevas regulaciones sociales, abren un abanico de posibilidades a los actores sociales, a la vez que les circunscriben el horizonte -que es diverso según el espacio social ocupado por ellos-.

En este entrecruce de relaciones se buscó indagar más precisamente la confluencia de componentes que participan en la elección de una carrera universitaria de profesorado, en la construcción de las representaciones ocupacionales del/la joven. Se buscó identificar en estas elecciones cómo se juega la construcción de futuro de nuevas generaciones en vistas a su inserción ocupacional, entendiendo que los habitus son duraderos en el tiempo, transmiten percepciones y valoraciones sociales históricamente construidas y, para el caso que aquí interesa, se despliegan en una condición social abruptamente diferente a la que se constituyó -se hace referencia a las transformaciones que el capitalismo tardío introduce en la condición humana-.

En esta tensión, -estar transitando una transformación en el campo de la economía, del trabajo, del conjunto de la sociedad-, se toman decisiones vin- 
culadas a la construcción de futuro, en este caso específicamente localizado en el campo ocupacional (que por cierto impacta y se ve impactado por los diferentes campos sociales), ¿qué es o cuáles son los aspectos que priman en el hecho de elegir una carrera universitaria de profesorado?

Desde este punto de partida, en el que se definieron como relevantes las relaciones que se producen entre los sujetos sociales -mayoritariamente jóvenes- la educación y el trabajo, -dadas las condiciones macro-estructurales que están conformando y tensando el tiempo socio-histórico-, se analizaron los sistemas de representaciones que los estudiantes tienen respecto de la educación, la docencia y el trabajo docente, buscando reconstruir algunas explicaciones vinculadas a las elecciones de esta carrera de cara al futuro.

Se identificó asimismo como estructurante de esta indagación, una dimensión institucional, localizada específicamente en la institución educativa que recibe a los estudiantes, la Facultad de Ciencias Sociales (FACSO) dependiente de la Universidad Nacional del Centro de la Provincia de Buenos Aires (UNICEN). Las marcas históricas, los mandatos fundacionales, los modos en que esta institución se constituye en el día a día, dieron cuenta de algunas percepciones y valoraciones que los estudiantes realizan a la hora de optar por inscribirse en una carrera de Profesorado y a posteriori durante el transcurso de la carrera.

Desde ese marco se han seleccionado dos aspectos reconocidos en ese trabajo para presentar en este artículo, que invitan a reflexionar y debatir entorno al ingreso a la Universidad Pública.

En principio, se presentarán algunas de las características identificadas en relación a aspectos se están jugando en la elección de la carrera de Profesorado universitario, atendiendo que estas carreras en buena medida están en franca vinculación -al menos curricular-, con las carreras de Licenciatura que se dictan en la sede académica.

En segundo lugar, se cambiará de foco para interrogar a la propia institución formadora sobre la percepción construida y compartida respecto de los estudiantes que eligen inscribirse en sus carreras. Aquí se pretende poner en debate las construcciones instaladas respecto de la referencia a "los puros", aquellos que llegan a la Facultad para inscribirse como estudiantes de un Profesorado, frente a los otros (¿impuros?) que llegan desde las licenciaturas y se matriculan en el Profesorado una vez que ya son estudiantes de la unidad académica.

\section{Primera parte}

El trabajo de investigación fue de carácter cualitativo y como herramientas metodológicas para la recolección de datos, se recurrió a *entrevistas en profundidad a ingresantes a profesorados de primera elección y de segunda elección; *entrevistas a estudiantes de profesorado de segunda elección; *dos 
talleres con estudiantes que asisten a la primera materia del área de la Formación docente, *un taller con estudiantes ingresantes a la FACSO y *entrevistas a informantes clave. También se utilizaron *fuentes de datos complementarias, como fueron el relevamiento realizado en un taller del Curso de Integración a la Vida Universitaria; material de seguimiento del Programa de articulación Polimodal Universidad (Informes, entrevistas a estudiantes y registros de reuniones); " registros informales de intercambios personales con estudiantes. Así mismo *archivos periodísticos del diario local fueron relevantes para la etapa de rastreo histórico. La recolección de datos se realizó entre febrero 2007 y agosto de 2008. Las entrevistas y los talleres se centran en relevar representaciones e imágenes de la ciudad vivida, el trabajo, la educación, la docencia, la enseñanza, las proyecciones socio-laborales.

A partir del trabajo de campo se relevó que los estudiantes en sus búsquedas de horizontes de futuro, están atravesados por construcciones sociohistóricas propias del estado de Social remontándose a un tiempo pasado -el de sus padres en sus inicios laborales y sus abuelos- en los que el trabajo era una conquista "personal" meritocrática, aunque la red social amplia abría oportunidades para quienes estuvieran en esta etapa.

La profundización del individualismo propio de estos tiempos, coloca a los sujetos en un campo de limitadas oportunidades y los carga de las responsabilidades de ser capaces (o no haberlo sido) de ubicarse "por las suyas" laboralmente. En este sentido, los estudiantes desde su posición de hijos, valoran las actividades y el esfuerzo de la familia, en algunos casos remontando hacia la historia parental y el modo en que superaron el campo social de sus abuelos, pero en general, desean "otra cosa" para ellos. Los estudiantes identifican la elección de una carrera de profesorado universitario como una oportunidad de trascender el tipo de ocupación que tienen los padres, vinculada a alcanzar una titulación universitaria, como posibilidad de despegar de un modo de subsistencia familiar, como alternativa de cambio social.

\section{Los estudiantes, la Facultad y el mundo del trabajo}

Si se toma como punto de partida caracterizar a los ingresantes a las diferentes carreras de la FACSO -se cursan licenciaturas en Comunicación Social, Antropología Social y Arqueológica y dos Profesorados-, se encuentran continuidades entre los datos, elementos que conformarían un continum, como es la localía, el que mayoritariamente son solteros, viven con su familia, son los primeros (ellos o sus hermanos/ primos) de la familia en llegar al nivel educativo superior. En el caso de los estudiantes de profesorado 2008, se auto-enclasan como de "clase media", "clase media baja" o bien "clase trabajadora" dando cuenta de un bienestar económico que la familia tuvo pero que se ha deteriorado. Desde su participación en el ámbito de la cultura, la Universidad se constituye 
en una oportunidad de mantener alguna continuidad con la participación social familiar o bien hacer un "salto social" hacia adelante.

Entre los que eligen una carrera de Profesorado, pueden identificarse dos grupos. Los ingresantes de primera elección y los de segunda elección.

Los de primera elección son aquellos que se llegan a la Facultad y se inscriben en un profesorado. Se identificaron dos características que marcan diferencia: la vinculación con el trabajo y la homogeneidad de edades. Así, se reconoce una inclusión de los estudiantes con el ámbito laboral (100\% trabajaban en el momento de la recolección de datos), a la vez que ese trabajo tienen una escasa vinculación con la carrera elegida. Además, la edad de los inscriptos es mucho mayor que la media de ingresantes. (En el año 2008, media, mediana y modo de los ingresantes a Profesorados fue de 28 años, mientras que la media del ingreso fue 21 años, según datos de la Secretaría Académica de la FACSO. Dto. Alumnos. 2008). En este sentido, la necesidad de contar con ingresos propios o de ayuda familiar, da cuenta de un sujeto que participa de una situación familiar que requiere de su aporte.

En el caso de los estudiantes de segunda elección, provenientes de las licenciaturas, y llegan al profesorado a posteriori, se identifica un aspecto llamativo: muchos de los estudiantes mantienen relaciones laborales con la propia institución educativa. Estos vínculos se materializan a través de becas (en programas propios de la Facultad o la Universidad), algunas formativas -docencia, investigación- pero otras se denominan de "contraprestación de servicios". Estas implican contratación de estudiantes para diferentes actividades.

Las transformaciones que se produjeron a partir de la implantación del modelo neoliberal, impactaron en los ámbitos laborales, de lo que la Universidad no quedó exenta. La flexibilización impactó más a los más débiles, en este caso a quienes inician sus primeros trabajos. Las becas de contraprestación de servicios, las pasantías, si bien son figuras que permiten al estudiante tener primeras experiencias rentadas (de muy bajos ingresos, al menos en esta Facultad) le retiran los beneficios que históricamente un auxiliar alumno tuvo (aportes jubilatorios, derecho a una obra social, antigüedad). Zona de riesgo, si se quiere, en términos de la trampa que se presenta al identificar el cierre de la carrera con la pérdida del ingreso económico o de visualizar a la Universidad y a la Facultad como "bolsa de trabajo" -prácticas muy favorecidas por las políticas clientelares de algunos tipos de gestión política-.

Asociado a esto, no pueden dejar de analizarse los modos en que las carreras fueron (o no) planificadas en vinculación al campo laboral y las acciones por las que las instituciones formadoras vinculan a los estudiantes con el campo laboral. La vulnerabilidad, por falta de red de contención social de los estudiantes se profundiza en la medida que la institución formadora se constituye desde un lugar "de encierro", dejando al actor sin conexiones con la realidad que está "tras sus muros" y que encontrará cuando esté solo, al egresar. 
Así, se presenta a continuación un relato registrado en una clase, a posteriori de una visita a una escuela de "contextos de encierro" - se trata de la escuela de una escuela primaria de adultos que funciona en una Unidad Penal de Sierra Chica, Olavarría, Pcia. de Bs As.- en la que un interno de la Unidad Penal expresa su desorientación y angustia por "el afuera" (quedaba en libertad en pocos días), ya que era muy incierta su posibilidad de encontrar trabajo (falto de educación y de una habilidad específica) y carecía de recursos personales o familiares para la subsistencia, y ahora además con antecedentes penales. En la mencionada clase se interroga a los estudiantes acerca de qué registros tenían de la visita a la escuela. Se presenta aquí un relato que tiene como protagonista a Mónica, una estudiante tesista de licenciatura. Ella es hija de una familia que migra de una pequeña localidad de la zona de Cuyo a Olavarría por trabajo; la empresa que emplea a jefe de familia quiebra en los '90. A pesar de quedar en muy malas condiciones económicas -el jefe de familia pasa a ser chofer transportista y la madre ama de casa, de una familia con cuatro hijos-, deciden permanecer en la localidad por las oportunidades educativas para los hijos (Taller 1). En esta etapa de su vida cursa el profesorado. Ante la pregunta de qué les resonaba a posteriori de la visita, ella manifiesta (con gran carga de angustia y por momentos su voz se vuelve con tono de enojo) que se siente igual que ese detenido que "esta por salir", como le pasa a ella "a nadie le preocupa lo que le pase" o "a dónde va a ir cuando salga". Esta expresión de sentimientos de desprotección, de falta de estructuras que desde la propia institución educativa articulen con el mundo del trabajo, la condición de fragmentación que se da entre el campo formativo y el laboral, y una brecha amplificada por la carencia de una red de relaciones que sostengan al sujeto en este traspaso de campos. Como el convicto a punto de egresar de la cárcel, la futura graduada se pregunta qué será de ella. Paradoja aparente, entre posiciones que teóricamente estarían muy distantes desde una mirada social que pondere a los sujetos por su escolaridad: un sujeto sin educación ni formación laboral, con antecedentes penales, y uno graduado de la Universidad. Parecerían lugares construidos por oposiciones, sin embargo los actores describen su vivencia personal frente al "egreso" de igual modo, cargado de angustia frente a la inserción laboral.

Por otro lado, no sólo emerge en esta escena la trama social familiar de los estudiantes, sino también la condición de reconocimiento y legitimidad que el título conlleva. Es bien diferenciada la posición de profesiones de origen tradicional que tienen campo propio que es reconocido y valorado socialmente, y aquellas otras que se hallan buscándolo, que necesariamente deben disputárselo a otros, replanteando de este modo la división del trabajo. Estas son profesiones "en vías de institucionalización" (Testa, J. 1997; Testa, J. Lorenzo, M 1998/1999, citado en Testa, J. y Spinosa, M.2008), como es el caso de la Comunicación Social. La posición de los egresados de estas últimas carreras, 
agrega a este escenario otros componentes con los que se complejiza aún más su incorporación al mundo laboral: las transformaciones en un mercado de trabajo, sujeto a reglas y condiciones que precarizan fuertemente la contratación; fuerte credencialismo y proliferación de ofertas de títulos de grado y postgrado (Testa J, 2003) como modo de generar valores diferenciales en el mercado externo que toma al diploma como moneda de cambio a la hora de definir contrataciones y promociones.

Es este el contexto que está mostrando, tal vez en el cierre de la carrera, pero que se sospecha desde el principio la escasez de espacios y la precaria condición en la que se encontrará al egresar. Es recurrente en los cursos de ingreso (CIVU) y en las promociones de carreras que se hacen desde la Facultad, la pregunta ¿de qué trabaja un Comunicador/un Antropólogo Social?, ¿dónde trabaja? Un trabajo como contratado o en calidad empleado, con bajos salarios, sin mayores garantías sociales y continuidad. Con una condición económica que no le permitirá afrontar el cursado de carreras de postgrado fuera de la ciudad, ya que implicaría afrontar no sólo los costos que estas carreras tienen desde la sanción de la Ley de Educación Superior, sino también la movilidad, estadía y ausencias a los lugares de trabajo que no contemplan licencias para esta actividad. Casi un imposible, si es que no se logra permanecer dentro de la Universidad, ahora como "Profesor" (aunque no haya hecho la formación como Profesor).

\section{Elegir una carrera de Profesorado}

Las preguntas que se plantearon al abordar el proceso de campo, aquellas que buscaban identificar si las matrices de aprendizaje familiares, que se entraman con la identidad de la localidad, con las historias familiares, y con los modos en que los sujetos aprenden a gestionar su propio conocimiento, intervienen en la elección de la formación docente, llevó a identificar algunos de los matices por los que la docencia es elegida.

Se reconoce que para quienes eligen estudiar una carrera docente, la FACSO es una oportunidad: por "estar en la ciudad", por ser "pública", porque "pueden trabajar". También, es una oportunidad de alcanzar un campo social diferenciado, al que escasamente o ningún miembro de su familia logró llegar. Y Olavarría, el lugar elegido para quedarse.

Una recurrencia inicial la señala como un campo profesional valorado, como salida "segura", componente residual de un modelo de sociedad salarial. Es vista como profesional, en la medida que es una formación universitaria, conllevando una distinción social que se estaría activando al llegar a un puesto de trabajo (vinculado al Estado, seguro, con ciertas garantías sociales residuales) y con un capital cultural de credencialidad que les amplia el campo social de la configuración que de la que ahora son parte.

Pudo identificarse a partir de las entrevistas la existencia de un sistema de creencias vinculadas a lo religioso, en particular al cristianismo, que relaciona 
en primer término las elecciones de ser profesor con la vocación, como elemento central a la hora de definir su inscripción a la carrera. El llevar la palabra, hacerse escuchar, influir para cambiar al otro se constituyen en descriptores de la tarea docente. Las experiencias por las que los estudiantes transitaron y construyeron estas proyecciones, se activan en la visión de sí mismos, en un aula, buscando influir en "los pensamientos" de sus alumnos. Ubican al "aula como lugar de peso", y a la escuela inmersa en problemáticas sociales, pero no como tarea resolverlas sino como "espacio de trabajo para" la toma de conciencia, que "es una forma de participar de esas soluciones", "un lugar para fomentar el cambio, pensar, sembrar conciencia". "No tanto hacer el cambio sino más bien fomentarlo". Se considera a la escuela como un "sitio estratégico" en el que permanece la "esperanza de cambio".

Esta intención de conquista de la zona más íntima de los sujetos por parte del educador, el pensamiento de los estudiantes, deja abierta una nueva veta para continuar en la indagación respecto de la institución formadora y las perspectivas que subyacen a sus propuestas (diseños curriculares y curriculum en acción), es decir si la formación para la tarea de enseñanza busca un docente "domesticador" o "emancipador."

Retomando, el sistema de creencias, podría explicar la "decisión" de permanecer próximos a los vínculos familiares. Se activa el sentido de "cuerpo" cristiano; la iglesia la conforman los miembros de la feligresía. La familia y los vínculos sostienen al sujeto, dan "raíces" que nutren a la vez que retienen. La mirada en el horizonte local estaría dando cuenta de este reconocimiento de pertenencia. El límite esta cerca, lo que no es para uno, desde un acto de resignación, una aceptación de "que las cosas son asi", hace que se busque al menos "algo chiquito" que de alguna satisfacción. Aceptar que esto es lo que le ha tocado, valorar lo que se tiene y "sacar lo mejor de sí" para gratificar y gratificarse.

Las marcas que dejaron los docentes a lo largo de las trayectorias escolares, dan cuenta de reconocimientos y distancias. La imagen residual de un docente respetado, con plenos derechos de realizar la tarea según su "ilustrado" criterio (al punto de ser legítimo ejercer la violencia) coloca al docente actual indefenso, frente a las transformaciones que se han ido dando, llevando a que la tarea se realice "para comer". Un desclasamiento del profesor, que ahora lo coloca como trabajador. La tarea docente puede dar cuenta de referir a sujetos que solo están interesados en un salario o aquellos que tienen "vocación" y se los reconoce ya que logran entrar al ámbito más privado de los sujetos, sus pensamientos. Estos últimos son los que revalorizan la tarea docente y se reconocen al retrotraerse al momento de la elección de carrera. 


\section{Segunda parte}

\section{Caracterizar la elección: de estudiantes "puros" a estudiantes de 1 ra. y 2 da. elección.}

El reconocimiento que se dará cuenta a continuación puede ubicarse en lo que comúnmente se denomina "la cocina de la investigación".

Un primer aspecto refiere a la denominación de los sujetos que conformaban en el inicio de la investigación, el universo de análisis. Esto se reconoció como un obstáculo que impactó en la definición del objeto de estudio, redefiniéndose en la instancia de este reconocimiento.

En principio la conformación del universo estaba definida en término de jóvenes y seguidamente, estos jóvenes eran supuestos como aquellos egresados de la escolaridad secundaria que se inscribían inmediatamente a una carrera de profesorado. En este primer foco, queda en evidencia rápidamente que la categoría jóvenes quedaba "incómoda" en la medida que los que se inscriben al profesorado el año en que se hace el relevamiento tienen como media, mediana y modo 28 años. Esta es una tendencia que se venía relevando desde hacía algunos años. En muchos casos los ingresantes provienen de otras formaciones preliminares; han dejado en algún momento una carrera y ahora intentan en esta nueva propuesta; están buscando una certificación ya que están trabajando desde su formación previa pero no poseen título habilitante, son algunas de las explicaciones que se relevan. Se explicita asimismo la imposibilidad de salir de la ciudad a estudiar y debe elegirse algo de lo que se ofrece en el horizonte de lo local. A partir de este reconocimiento, la decisión fue cambiar la denominación de este universo, de pensar en jóvenes a denominarlos estudiantes. Queda en tensión así, la opacidad de la mirada sobre quiénes son estos que están llegando a la Facultad demandando por una carrera de profesorado.

Al mismo tiempo se dejan al descubierto ciertos supuestos conceptuales que limitaban/ sesgaban el recorte del objeto, ya que circunscribían la categoría joven a la categoría etaria, y asimismo, que las elecciones son móviles, se siguen dando a lo largo de la vida de los sujetos, se redefinen y actualizan.

Como segundo elemento que se hace visible en esta etapa reflexiva es el reconocimiento de una "pantalla"-aspectos (dichos y no dichos) que sostienen y refuerzan modos de hacer y de explicar "cómo son aquí las cosas", opacando componentes simbólicos e ideológicos que conforman la cultura institucional- que se identifica cuestionando al lenguaje, componente de la cultura institucional. En la jerga de la institución, más específicamente al interior del propio Profesorado, a los estudiantes que llegan a la Facultad y se inscriben a un profesorado, se los denomina "los puros". Esta nominación no es exclusiva de esta casa de estudios, ya que en otros ámbitos universitarios de similares características, este término se utiliza del mismo modo. 
Hablar de puros implica también suponer su par puesto, los "impuros". Es decir, diferenciar aquellos que llegan a la elección de profesorado porque "naturalmente" parecería que querían / iban a ser profesores, casi como una condición intrínseca, frente a otros que se presentan como "advenedizos", ya que es la licenciatura lo que los ha convocado a la Universidad. Se revela bajo esta nominación -los puros- una categoría esencialista de identidad, cristalizada, ya que parte de ciertas presunciones en las que una condición que se despliega -ser profesor- era algo inherente a los sujetos. Perviven ideas de que alguien es docente casi "por naturaleza", tiene en esencia ese destino. Pero hasta esta instancia de la investigación esto no había sido advertido. ¿Por qué? Porque en un primer recorte se había definido tomar exclusivamente a aquellos que llegaban por primera elección -puros- y, como sujetos jóvenes, debían venir del secundario directamente, dejando afuera del universo a los estudiantes que llegaban al profesorado desde las licenciaturas.

En realidad a partir de este reconocimiento es que se definen nuevas categorías para tomar a los estudiantes. Se define trabajar con ingresantes y con estudiantes ya en carrera y ahí se los nombra de este modo, de "1ra. y de 2da. elección."

En esta nueva nominación, el reconocimiento y necesidad de incluir a "los de 2 da. elección", se explicita una vinculación entre la elección y la categorización que se hace de los estudiantes, con el mandato fundacional no dicho de la FACSO. Siguiendo a Lidia Fernández (1994, 1996), se reconoce que la historia institucional y el origen de las instituciones educativas "tienen una especial significación como expresión de su potencia para progresar hacia el futuro". Así, (...) "es posible trabajar con la hipótesis de que el mandato social es efectivamente un doble mandato y como tal encierra una paradoja" (Fernández, L. 1996: 108). En sus mandatos explícitos y públicos se enlaza con una utopía y se expresa en fines y objetivos formales. En sus aspectos ocultos alude a un imperativo no explícito, relacionado con el lugar que los egresados tienen reservado en la estructura socioeconómica y en la distribución del poder político consecuente.

Cabe señalar que buena parte de los estudiantes de profesorado fueron y son de 2 da elección. Dejar afuera a este grupo era funcional a seguir opacando elementos fundantes no dichos de las carreras: dar salida a un gran grupo de estudiantes que no lograban hacer sus tesis de licenciatura, a través de la puerta del fondo, los profesorados. Así, Rosana Sosa (2006:187) explicaba que

(...) inicialmente, la creación del profesorado, lejos de significar una afluencia de inscriptos nuevos a la institución, se consolidó con la presencia de estudiantes que cursaban otras carreras en la FACSO. Desde los primeros momentos, la matriz fundacional de la nueva carrera nos muestra un primer atributo: el profesorado es una oferta educativa nueva para los alumnos de siempre. 
Este reconocimiento, hacia el interior del proceso de investigación permitió desocultar categorías implícitas que "seleccionan" y "clasifican". Incluir/excluir a un grupo no solo importante sino que en muchos años ha sido mayoritario en el número de ingresantes, aquellos que llegan a una carrera provenientes de la licenciatura, mostraba/ocultaba en parte el revés de la trama. Estos estudiantes, desde una construcción esencialista de las identidades que circula entre los docentes de los profesorados, son vistos como "congelados" en una identidad que convierte a esta segunda opción como impura, ya que no estaba en su esencia ser docentes, por lo que se invisibilizaban.

\section{Otra línea de urdimbre, para seguir tejiendo.}

En las Facultades en las que se ofrecen carreras de licenciatura en campos que tienen un objeto de conocimiento difícil de "localizar" con claridad en un puesto de trabajo, se reconoce a los profesorados como un ámbito formativo que cuenta con una clara inserción, especialmente en el sistema educativo en sus diferentes niveles y jurisdicciones. La tarea profesional del licenciado en estas carreras es un desafío a construir, a inventar, que requiere de un actor social que pueda asumir esta búsqueda con autonomía, que se anime y tenga herramientas para hacerlo.

Como contraparte, la docencia es un territorio conocido (al menos desde el lugar de estudiante), organizado, seguro, que da ciertas garantías sociales. Los componentes socio-familiares y las identidades locales están aportando a la lectura de la tarea del profesor como vocación/trabajo/profesión. Posiciones éstas que se superponen, alternan o priman según el momento y la lectura que los actores hacen de la tarea pero con una fuerte presencia de marcas religiosas en sus trayectorias que componen por momentos representaciones de la tarea muy asociadas a la evangelización.

En un trabajo anterior, que tomaba como referentes estudiantes de diferentes profesorados de la UNICEN (Umpierrez, A., 2008c: 10), se señalaba haciendo referencia a este grupo de estudiantes que llegan a los profesorados provenientes de las licenciaturas:

La carrera se elige por la disciplina; luego, el enseñar la disciplina se identifica como oportunidad y diferenciación en la inserción laboral. En el recorrido de formación docente específica, y en particular en las experiencias que los colocan en vinculación con la práctica, se valora y elige la docencia como lugar para permanecer. La elección de "pasar" por la formación se desliza a una decisión "quedarse" en la enseñanza. Lo que era una "puerta de salida", se convierte en una "puerta de entrada" a un campo profesional que no se sospechaba de esas dimensiones y que se quiere explorar. 
Asimismo, puede asegurarse que los jóvenes que llegan a la FACSO ven en la educación superior universitaria una oportunidad de cambio social, como condición para afrontar el mundo que les toca vivir y que la familia apuesta. Más allá del cambio de condición económica, sigue siendo una proyección familiar que remite a un imaginario social que aún coloca en la educación la oportunidad de "salto social" (al menos cultural). En este sentido, los estudiantes se están visualizando como graduados e identifican allí una oportunidad de incorporarse a un campo social diferenciado, a partir de fuerzas de juego, en términos de Norbert Elías (1999), que se activan con la titulación y modifican la configuración social de la que son parte.

Las reflexiones que se han planteado hasta aquí apuestan también a poner en tensión las representaciones de los profesores universitarios respectos de aquellos que eligen formarse en la docencia. Es sabido que al interior de la Universidad se siguen sosteniendo disputas históricas, como que para enseñar en la universidad no hay que tener un título docente; espacios en los que las carreras de Profesorados son vistas como de menor valor y "fáciles" respecto de las licenciaturas, entre otras. En muchos casos los egresados de licenciatura eligen trabajar como profesores (en la Universidad o en otros niveles del sistema educativo), sin pasar por la formación docente. Este es otro pliegue de la trama, que no se aborda aquí pero que se señala, ya que esta decisión de "ser profesor sin pasar por la formación" indica otras pistas, vinculadas al valor asignado al conocimiento disciplinar por sobre la especificidad de la enseñanza.

Es central en momentos en que se están debatiendo estándares de acreditación de los Profesorados Universitarios como carreras de interés público, revisar las propuestas curriculares, académicas, de transferencia, atendiendo no solo a las definiciones que puedan hacerse desde ámbitos académicos internacionales respecto de los campos disciplinares. (A partir de la Resolución 50/2010 del Ministerio de Educación de la Nación, que declara incluido en la nómina del artículo 43 de la Ley № 24.521 el título de Profesor Universitario, los Profesorados de todo el país deberán acreditarse como allí se estipula El artículo 43 de la Ley de Educación Superior establece que los planes de estudios de carreras correspondientes a profesiones reguladas por el Estado, cuyo ejercicio pudiera comprometer el interés público, poniendo en riesgo de modo directo la salud, la seguridad, los derechos, los bienes o la formación de los habitantes, deben tener en cuenta - además de la carga horaria mínima prevista por el artículo 42 de la misma norma- los contenidos curriculares básicos y los criterios sobre intensidad de la formación práctica que establezca el Ministerio de Educación en acuerdo con el Consejo de Universidades).

Es relevante también identificar y ponderar quiénes son los que llegan, los modos en que cada unidad académica actualiza y desarrolla sus propuestas, cuáles y cómo se dan las vinculaciones con el mundo del trabajo y analizar reflexivamente las prácticas que se sostienen desde los núcleos rígidos de creencias (Gravano, A. 1992) de las culturas institucionales. 


\section{Referencias bibliográficas}

- Baumann, Z. (1999) La Globalización. Consecuencias humanas. Bs. As., Argentina. FCE.

- Baczko, B. (1991) Los imaginarios sociales. Memorias y esperanzas colectivas., Bs. As. Argentina. Ediciones Nueva Visión.

- Bourdieu, P. (1987) Los tres estados del capital. Revista Sociológica. №5. Año 2. Otoño México, Universidad Autónoma Metropolitana. (1997). Capital cultural, escuela y espacio social. México. Siglo XXI. (2004). Miseria del mundo. Bs As Argentina. FCE. (2007). El sentido práctico. Bs. As. Argentina, Siglo XXI. Chaves, M. (2005). Juventud negada y negativizada. Representaciones y formaciones discursivas vigentes en la Argentina contemporánea Revista Última Década №23, CIDPA Valparaíso. Chile. PP. 9-32.

- $\quad$ Elias, N.(1999). Sociología fundamental. Barcelona. Gedisa

- $\quad$ FACSO. UNICEN.2003, 2004, 2005 y 200 Informe ¿Quienes son los ingresantes? Programa de Articulación Polimodal/Universidad. Mimeo.

- Fernández, L. (1994). Instituciones educativas. Dinámicas institucionales en situaciones críticas, Bs As. Argentina, Paidós.

- Giménez, G. (1997). "Materiales para una teoría de las identidades sociales".

- http://www.insumisos.com/lecturasinsumisas/Teoria\%20de\%20las\%20identidades.pdf. Visitada 20/12/07

- $\quad(\mathrm{S} / \mathrm{F})$ "La cultura como identidad y la identidad como cultura" http://www.pucp.edu.pe/ ridei/pdfs/laculturacomoidentidadylaidentidadcomoculturagilbertogimenez.pdf Visitada el $18 / 12 / 07$

- Gravano, A. (1992) "Antropología Práctica: muestra y posibilidades de antropología organizacional": Publicado en Antropología y Ciencias Sociales, ( $N^{\circ} .1$, año 1), Buenos Aires; SIN 0327-6627; Pág. 95-126. FACSO.

(comp.) (2005). Imaginarios sociales de la ciudad media. Argentina. UNICEN.

- $\quad$ Chaves, M. "Investigaciones sobre juventudes en Argentina: estado del arte en ciencias sociales". Con la colaboración de María Graciela Rodríguez y Eleonor Faur. Informe para el Proyecto: Estudio Nacional sobre Juventud en la Argentina. Dirigido por Eleonor Faur. IDAES-UNSAM-DINAJU. Buenos Aires. Mayo 2006. 93 pp.

- Kaminsky, G. (1994). Dispositivos institucionales. Democracia y autoritarismo en los problemas institucionales. 2da edición. Bs As. Argentina. Lugar editorial.

- Mons, A. (1994). La metáfora social. Imagen, territorio, comunicación. Bs. As. Argentina, Nueva Visión.

- $\quad$ Ortiz, R. (1998a). Los artífices de una cultura mundializada. Fundación social. Siglo del Hombre Editores. Santa Fé de Bogotá, DC. Colombia.

(1998b) Otro territorio. Colombia, Convenio Andrés Bello.

Reguillo Cruz, R. (2000). Emergencia de culturas juveniles. Estrategias del desencanto. Bs. As. Argentina, Tesis Norma

(2006). "Políticas de la mirada. Hacia una antropología de las pasiones contemporáneas". En Dussel, I y Gutierrez, D. Comp. Educar la mirada. Políticas y pedagogías de la imagen. Argentina. Manantial, FLACSO y Fundación OSDE.

(2007). "Ciudad y Comunicación. Densidades, Ejes y Niveles". Revista Académica de la Federación Latinoamericana de Facultades de Comunicación Social - Mayo Agosto 2007 Nro. 74- en http://www.dialogosfelafacs.net 
- $\quad$ Remedi Allione, E. (2004). Instituciones educativas. Sujetos, historia e identidades. México. Plaza y Valdés editores.

- Sosa, R. (2006) "Los docentes y la tarea de buscar trabajo. Una estrategia para identificarse y diferenciarse. El caso de los graduados de los profesorados universitarios de la FACSO UNICEN". Cuadernos de educación. Año IV Número 4. Córdoba, Argentina. Pp. 187

- $\quad$ Sosa, R. y Umpierrez, A. (2004). "La opacidad del ingreso: ¿Qué demanda el ingresante que la Facultad aún no ve? Curso de Integración a la Vida Universitaria de la Facultad de Ciencias Sociales. UNICEN. Algunas reflexiones del trabajo realizado". En I Encuentro Nacional La problemática del ingreso a Carreras de Humanidades, Cs. Ss. y Artes en las universidades públicas. UNC, Córdoba. Inédito.

- Testa, José. Spinosa, Martín. (2008) Las expectativas profesionales de los alumnos próximos a graduarse de la Licenciatura de Cs. de la Educación de la UNLu. UNLu. Argentina.

- Umpierrez, A. (2008a). Representaciones sobre la docencia en relación con el proceso de incorporación a la formación de jóvenes ingresantes universitarios. KAIROS. Revista de Temas Sociales. (Año 12. № 21. Junio de 2008) ISSN 1514-9331. URL: http://www. revistakairos.org. Proyecto Culturas Juveniles Urbanas. Publicación de la Universidad Nacional de San Luís.

(2008b). Actores de instituciones. Campo de juego en la construcción de identidades docentes. En Chapato, M.E y Errobidart, A. (comps.) Hacerse docente. Las construcciones identitarias de los profesores en sus inserciones profesionales. Miño y Dávila. Bs As. Argentina. P.115-132

(2008c). "La elección de una carrera de profesorado, de "puerta de salida" a "puerta de entrada" a un campo socio-laboral". Manuscrito presentado para su publicación. FACSO. UNICEN.

(2009). El acceso a una carrera universitaria de profesorado en una universidad regional: entre elecciones y oportunidades de un campo ocupacional, determinaciones y autonomías, sujetos y contextos. Tesis de Maestría no publicada. Facultad de Ciencias Humanas. UNICEN.

(2010a). "Imaginarios, identidad local y elección de carrera: la ciudad como horizonte". Manuscrito presentado para su publicación.

(2010b). "Estar en el borde y tratar de no caer. Jóvenes que eligen la docencia universitaria como campo socio-laboral". Revista El Cardo No 11- Facultad de Ciencias de la Educación. Universidad Nacional de Entre Ríos. Área Didáctica. Verano 2010. Pp.138-155

- Vila, P. (2001). "Identidades culturales y sociales" en Di Tella, T. Diccionario de Ciencias Sociales y políticas. Emece Buenos Aires, Argentina.

- Zibecchi, C. (2002). "Universidades argentinas en un contexto neoliberal: un análisis centrado en la experiencia y las percepciones de sus estudiantes". Informe final del concurso: La educación superior en América Latina y el Caribe. Redefinición de las fronteras entre lo público y lo privado. Programa Regional de Becas CLACSO. 2002. Extraído el 1 de setiembre de 2008 desde http://bibliotecavirtual.clacso.org.ar/ar/libros/ becas/2002/edu/zibecchi.pdf 